\title{
Soft landing of size selected clusters in rare gas matrices
}

\author{
J.T. Lau ${ }^{1}$, H.-U. Ehrke ${ }^{2}$, A. Achleitner ${ }^{2}$, and W. Wurth ${ }^{1}$ \\ ${ }^{1}$ Universität Hamburg, Institut für Experimentalphysik, Luruper Chaussee 149, D-22761 Hamburg \\ E-mail: wilfried.wurth@desy.de \\ ${ }^{2}$ Technische Universität München, Physik Department E20, D-85747 Garching
}

Received November 10, 2002

\begin{abstract}
Soft landing of mass selected clusters in rare gas matrices is a technique used to preserve mass selection in cluster deposition. To prevent fragmentation upon deposition, the substrate is covered with rare gas matrices to dissipate the cluster kinetic energy upon impact. Theoretical and experimental studies demonstrate the power of this technique. Besides STM, optical absorption, excitation, and fluorescence experiments, $x$-ray absorption at core levels can be used as a tool to study soft landing conditions, as will be shown here. X-ray absorption spectroscopy is also well suited to follow diffusion and agglomeration of clusters on surfaces via energy shifts in core level absorption.
\end{abstract}

PACS: 61.46.+w, 87.64.Ni, 36.40.Cg, 36.40.Qv

\section{Introduction}

Clusters of atoms or molecules are fascinating objects to study the evolution of electronic [1-3], magnetic [4-7] or chemical [8-10] properties from isolated atoms to bulk matter. Many types of cluster sources [11-14] have been developed and adapted to investigate a wide range of physical phenomena $[15,16]$. A cluster experiment typically consists of a cluster production stage with mass selection and of a stage to probe the clusters' physical properties, either in free cluster beams or after cluster deposition.

To study the size dependence of any physical property of clusters, size selection is a prerequisite. In gas phase experiments on cluster beams, cluster ions can simply be size selected in electric (quadrupole) or magnetic (dipole) fields. To illustrate this, a mass spectrum of positively charged iron clusters mass separated in a magnetic dipole field is shown in Fig. 1. These iron clusters were generated by sputtering [17] of a high purity iron target with a $28 \mathrm{keV} \mathrm{Xe}^{+}$beam $[6,7,18]$. As can be seen in Fig. 1, size selected cluster current densities typically range from some $\mathrm{pA}$ to a few $\mathrm{nA}$ per $\mathrm{mm}^{2}$, depending on cluster size and material.

In cluster deposition experiments, however, not only size selection of the incoming cluster beam, but also the preservation of the preselected cluster size upon and after deposition of the clusters is crucial.
The incoming cluster beam of uniform size should not be transformed into a distribution of different cluster sizes on the substrate surface. Therefore, cluster deposition conditions have to be chosen under which no fragmentation, implantation, or agglomeration of the clusters occurs. For this purpose, the soft landing technique has been developed by several groups, both experimentally [19-25] and theoretically [26-32]. As a result, this technique is now well established. The principal idea of soft landing is to put the clusters down onto substrates as gently as possible. This in-

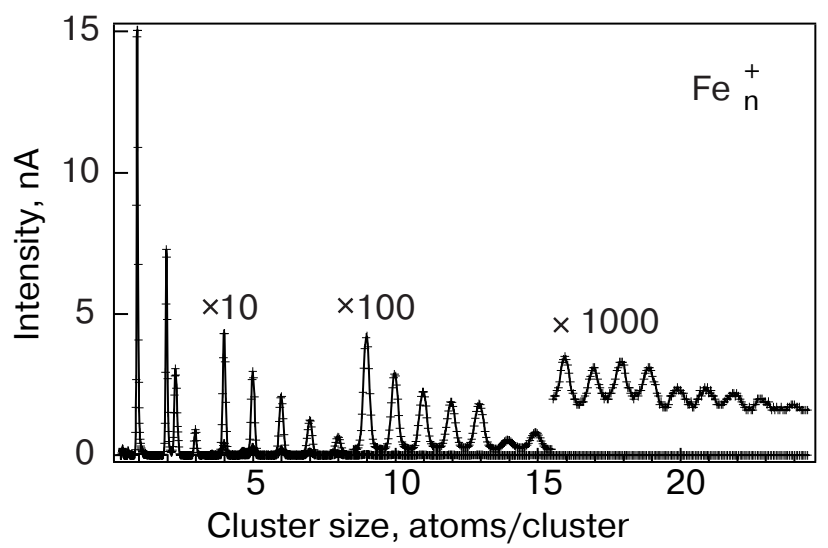

Fig. 1. Mass spectrum of positively charged iron clusters produced with a sputter source and measured on a detector area of $3 \mathrm{~mm}^{2}$. 


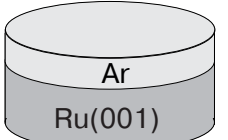

Ar adsorption

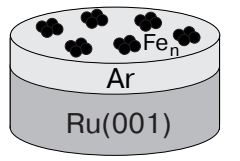

cluster deposition

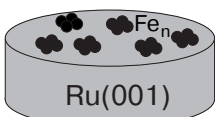

Ar desorption
Fig. 2. Schematic representation of the soft landing process: Adsorption of rare gas multilayers as a buffer layer on the substrate (left), cluster deposition at low kinetic energy into the rare gas buffer (center), cluster sample after rare gas desorption (right).

cludes deposition at low kinetic energy as well as deposition into soft matrices or buffer layers preadsorbed on the substrate. In most cases, these buffer layers will be rare gas multilayers adsorbed on the substrate material at low temperatures. After cluster deposition, the buffer layer will be desorbed from the sample surface by heating to above the monolayer desorption temperature. This allows to investigate the clusters directly on the substrate surface. Alternatively, the properties of mass selected clusters can be studied inside the rare gas matrix [25]. In Fig. 2, the idea of soft landing is illustrated schematically.

\section{The soft landing process}

The purpose of soft landing is to gently dissipate the clusters' kinetic energy in the deposition process. In soft landing of size selected clusters, clusters are size selected in the gas phase, decelerated to a few eV per cluster atom and deposited into chemically inert buffer layers which have been prepared on the substrate. Bond energies in metal clusters [33,34] are typically on the order of a few eV. By depositing a decelerated cluster beam with kinetic energies typically of some few $\mathrm{eV}$ per cluster atom into rare gas multilayers, the surplus kinetic energy will be dissipated by energy transfer into the matrix and ultimately by rare gas desorption. This process has been studied experimentally [19-25] and theoretically [26-32] in detail. Molecular dynamics (MD) simulations [26,27] of the energy dissipation processes provide insight into the different stages of slowing down the impinging clusters and redistributing their initial kinetic energy.

When colliding with the matrix or rare gas buffer, the kinetic energy of the clusters is converted to vibrational energy, resulting in a rapid heating of the clusters. Heating rates are on the order of $10^{15} \mathrm{~K} / \mathrm{s}$ and lead to superheated clusters. In the MD simulations [26,27], the cluster temperature roughly doubles when argon is used for soft landing instead of neon, and again when xenon is used instead of argon. The clusters cool down via heat transport into the sur- rounding matrix and via desorption of rare gas atoms. Cooling rates are lower than heating rates and are on the order of $10^{13}-10^{14} \mathrm{~K} / \mathrm{s}$.

As has been shown in thorough experimental investigations [19-22,24], the fragmentation rate of small metal clusters can be reduced to about $10 \%$ by using proper soft landing conditions. From optical absorption and excitation experiments [20,21,24] on clusters soft landed in rare gas matrices it is known that the efficiency of the soft landing process and the fragmentation rate of the clusters depends on the nature of the rare gas matrix. As discussed above, this is also known from theoretical predictions [26,27]. The lighter the adsorbed rare gas, the softer the buffer layer [20,21], which in turn leads to a more efficient suppression of cluster fragmentation. Since adsorption temperatures decrease with decreasing mass of the rare gas atoms, sample cooling down to at least $15 \mathrm{~K}$ is desirable for adsorption of argon buffer layers. Low temperatures are also desirable to minimize cluster diffusion and agglomeration on the substrate surface [35,36] after cluster deposition.

It has also been demonstrated [24] that the fragmentation rate depends on the initial kinetic energy of the clusters and on the binding energy of the cluster atoms. The higher the binding energy, the lower the fragmentation rate for otherwise identical conditions [24]. However, even after cluster deposition into rare gas matrices at a kinetic energy of $50 \mathrm{eV}$ per atom there is a non-negligible fraction of clusters that have not fragmented [24]. MD simulations [32] explain this in terms of recombination: While clusters will gain vibrational energy and may break up upon impact, they are also mobile and can recombine while the clusters and the surrounding matrix are still hot.

STM studies [22] on soft landed clusters also have shown the power of the soft landing technique. Furthermore, they reveal that most of the soft landed clusters form highly symmetric equilibrium structures on the substrate surface. Small clusters tend to form two-dimensional structures on a surface [22,37].

Even though soft landing can be used to preserve size in cluster deposition experiments, the geometry of deposited clusters will differ from the geometry of gas phase clusters due to the interaction with the substrate $[38,39]$. In the present study, two-dimensional structures can be assumed for the deposited clusters because of their small size.

\section{X-ray absorption experiments on soft landed metal clusters}

Soft landing can not only be studied in STM $[19,22]$ or fluorescence $[20,24]$ experiments, but also in x-ray absorption (XAS) studies. In x-ray absorp- 
tion, electrons from a core level are promoted into unoccupied states above the Fermi level. XAS is sensitive to the local electronic order and the chemical environment of the excited atom: Different species yield discernible fingerprints. Furthermore, XAS is an element specific probe, and it is sensitive to submonolayer quantities of adatoms and clusters on surfaces.

The experimental setup $[6,37,40]$ used for cluster deposition consists of a sputter source with mass selection and ultra-high vacuum (UHV) cluster deposition under soft landing conditions. The UHV chamber is equipped with all standard tools necessary for substrate surface preparation. A Ru(001) single crystal surface precovered with a $(2 \times 1) \mathrm{O}$ superstructure was used as a substrate for cluster deposition to reduce the cluster-substrate interaction. Other than for chromium clusters on $(2 \times 1) \mathrm{O} / \mathrm{Ru}(001)$ [37] that are highly sensitive to oxidation, no indication of oxidation was found for iron clusters. For in situ sample preparation, the $\mathrm{Ru}(001)$ surface was cleaned after sputtering by repeated cycles of heating to $1450 \mathrm{~K}$ in UHV and oxygen dosage while cooling down the sample. The $(2 \times 1) \mathrm{O} / \mathrm{Ru}(001)$ layer was prepared just before cluster deposition by heating the sample to $1450 \mathrm{~K}$ and cooling it down while dosing oxygen onto the $\mathrm{Ru}(001)$ surface. Surface quality was checked with XAS, low-energy electron diffraction (LEED) and Xe thermal desorption spectroscopy (TDS). The $\mathrm{x}$-ray absorption experiments were carried out at the synchrotron radiation sources BESSY I (HE-PGM3) and BESSY II (U49/1-PGM). The photon energy scales are calibrated relative to each other with the absorption spectra of stainless steel used as a reference.

Soft landing and fragmentation was studied for small clusters since for a given kinetic energy the kinetic energy per cluster atom is larger the smaller the cluster. The binding energy per atom, on the other hand, depends on, but does not decrease significantly, with cluster size [33,34]. Therefore, fragmentation upon deposition is most likely to be expected for small rather than for large clusters, and soft landing conditions are crucial for deposition of small clusters.

Small iron cluster XAS is shown in Fig. 3, where $2 p_{3 / 2} \rightarrow 3 d\left(L_{3}\right)$ absorption spectra of mass selected iron clusters on $(2 \times 1) \mathrm{O} / \mathrm{Ru}(001)$ are given for different soft landing conditions [41]. In the lower panel of Fig. 3, $\mathrm{Fe}_{1}, \mathrm{Fe}_{2}$, and $\mathrm{Fe}_{3}$ clusters were deposited into approx. 10 layers of argon, which was adsorbed on the $(2 \times 1) \mathrm{O} / \mathrm{Ru}(001)$ surface at less than $20 \mathrm{~K}$ prior to cluster deposition. Acceleration and retardation voltages were set to decelerate the cluster ions to less than $1-2 \mathrm{eV}$ per atom. Since the energy distribution of clusters produced in a sputter source $[42,43]$ is centered



Fig. 3. X-ray absorption at the $L_{3}$ edge of mass selected iron clusters on $(2 \times 1) \mathrm{O} / \mathrm{Ru}(001)$ for different soft landing conditions.

about 5-10 eV, with a width of approx. 5-10 eV, the potential applied to the sample was set to $11 \mathrm{~V}$ higher than the acceleration voltage. This allows only clusters with an initial kinetic energy of more than $11 \mathrm{eV}$ to reach the sample, and leads to a kinetic energy of less than 1-2 eV per atom even for small clusters. The equivalent coverage of iron atoms on the $(2 \times 1) \mathrm{O} / \mathrm{Ru}(001)$ surface is approx. 0.05 monolayers in all three cases.

The $L_{3}$ absorption edges of iron clusters in the lower panel of Fig. 3 clearly show different absorption energies. There is an energy shift in the $L_{3}$ edge absorption of about $0.5 \mathrm{eV}$ to higher energy in going from $\mathrm{Fe}_{1}$ to $\mathrm{Fe}_{2}$ and of about $0.8 \mathrm{eV}$ to lower energy in going from $\mathrm{Fe}_{2}$ to $\mathrm{Fe}_{3}$. The absorption spectra of these clusters were taken as deposited, at $20 \mathrm{~K}$ with the argon layer still present on the sample. 
This energy shift in iron $L_{3} \mathrm{x}$-ray absorption is a hint at different electronic structures of the cluster species on the surface that can be observed after the clusters have been deposited into argon multilayers with a kinetic energy of less than $1-2 \mathrm{eV}$ per cluster atom. Different cluster species with different electronic structure yield different $L_{3}$ x-ray absorption spectra. From the difference in the absorption spectra it can be deduced that soft landing conditions in this case are fulfilled and no cluster fragmentation occurs. The kinetic energy of the clusters is successfully dissipated by the argon buffer layers.

For comparison, in the upper panel of Fig. 3 x-ray absorption spectra of $\mathrm{Fe}_{3}$ and $\mathrm{Fe}_{6}$ clusters are shown which were deposited onto the $(2 \times 1) \mathrm{O} / \mathrm{Ru}(001)$ substrate that was covered with only one monolayer of argon atoms. The kinetic energy of the clusters again was 1-2 eV per cluster atom. After cluster deposition, the argon layer was desorbed from the $(2 \times 1) \mathrm{O} / \mathrm{Ru}(001)$ surface by heating to $100 \mathrm{~K}$. The spectra were taken at $20 \mathrm{~K}$. Two main features distinguish these spectra from the ones shown in the lower panel of Fig. 3. Both spectra in the upper panel look very similar and, in contrast to the clusters deposited into argon multilayers, exhibit one peak with a shoulder at the low energy tail. From the structure and energetic position of the absorption line it can be inferred that not only one but at least two different cluster species exist on the surface. Under these conditions, soft landing clearly fails. $\mathrm{Fe}_{3}$ and $\mathrm{Fe}_{6}$ clusters are fragmented or implanted if only a monolayer of argon is used as a buffer layer for cluster deposition.

\section{Cluster diffusion and agglomeration}

To further test our finding that soft landing in argon multilayers preserves the initial cluster size, iron adatoms, dimers and trimers deposited under soft landing conditions were annealed stepwise to room temperature. After annealing, the sample was cooled down again to $20 \mathrm{~K}$ for the measurements. Again, the size selected clusters were deposited into approx. 10 layers of argon preadsorbed on the $(2 \times 1) \mathrm{O} / \mathrm{Ru}(001)$ surface. These argon multilayers were desorbed from the sample surface after heating to $100 \mathrm{~K}$. For every cluster deposited, there are also spectra taken prior to argon desorption.

In Fig. 4, x-ray absorption spectra of iron adatoms on $(2 \times 1) \mathrm{O} / \mathrm{Ru}(001)$ taken at $20 \mathrm{~K}$ with argon still present, and after successive anneal steps to 100, 150, 200 , and $300 \mathrm{~K}$ are shown.

Annealing to $100 \mathrm{~K}$ does not significantly change the XAS line shape and position, although the $L_{3}$ line is slightly broadened. After annealing to $150 \mathrm{~K}$, how-

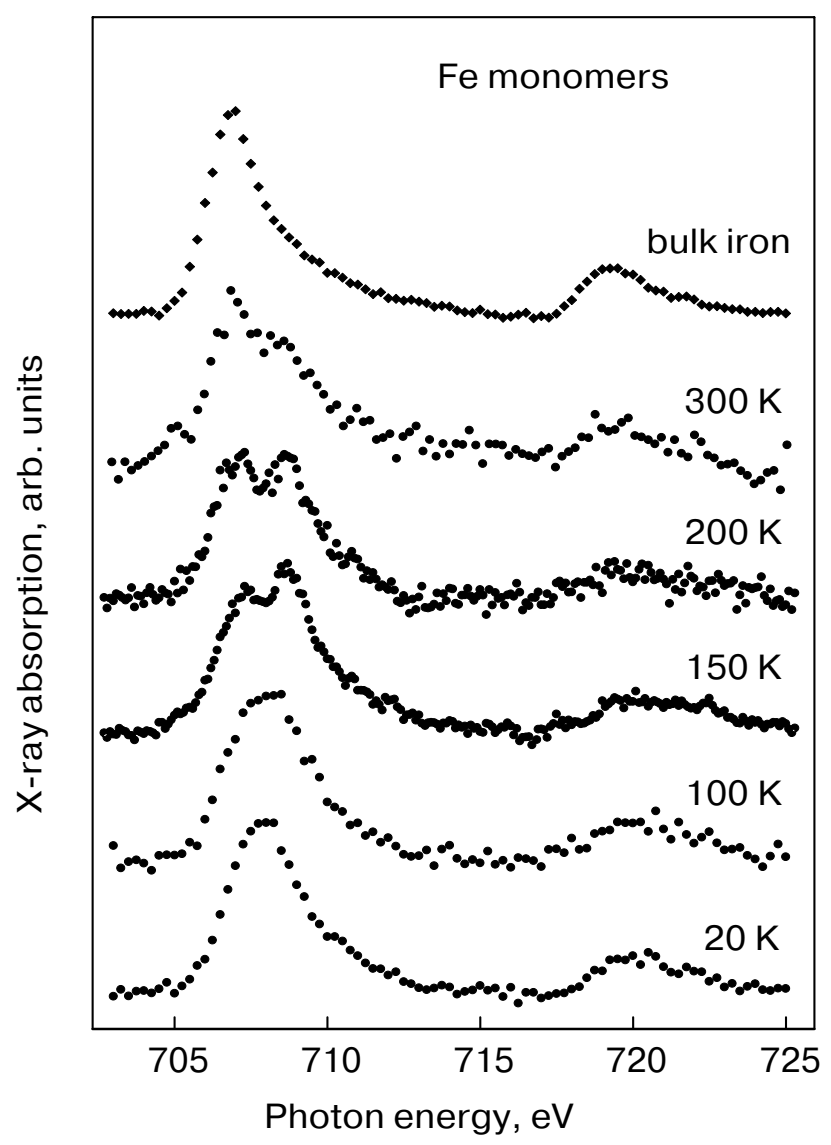

Fig. 4. $L_{2,3} \mathrm{x}$-ray absorption spectra of iron adatoms on $(2 \times 1) \mathrm{O} / \mathrm{Ru}(001)$ for different anneal temperatures. A step edge has been subtracted from all spectra [41].

ever, a double structure evolves in the $L_{3}$ line that is even more pronounced after annealing to $200 \mathrm{~K}$. At $300 \mathrm{~K}$, the first peak at approx. $707 \mathrm{eV}$ is already stronger than the second peak at approx. $709 \mathrm{eV}$. This first peak corresponds nicely to the $L_{3}$ line of a bulk iron sample, also given in Fig. 4.

This evolution of the iron $L_{3}$ line can be interpreted in terms of diffusion and agglomeration of the iron adatoms. After deposition, the only species on the $(2 \times 1) \mathrm{O} / \mathrm{Ru}(001)$ surface is iron atoms. Soft landing conditions are sufficient to inhibit implantation, and the coverage is low enough to rule out agglomeration. Obviously, fragmentation cannot occur for single atoms. Upon annealing to $150 \mathrm{~K}$, adatom diffusion leads to agglomeration and formation of iron islands on the $(2 \times 1) \mathrm{O} / \mathrm{Ru}(001)$ surface, as can be deduced from the evolution of a double peak at the $L_{3}$ absorption line. Since the low energy peak around $707 \mathrm{eV}$ corresponds with the $L_{3}$ line of bulk iron, it can be ascribed to larger islands of iron atoms that have formed. After annealing to $300 \mathrm{~K}$, most of the iron atoms on the $(2 \times 1) \mathrm{O} / \mathrm{Ru}(001)$ surface form these 


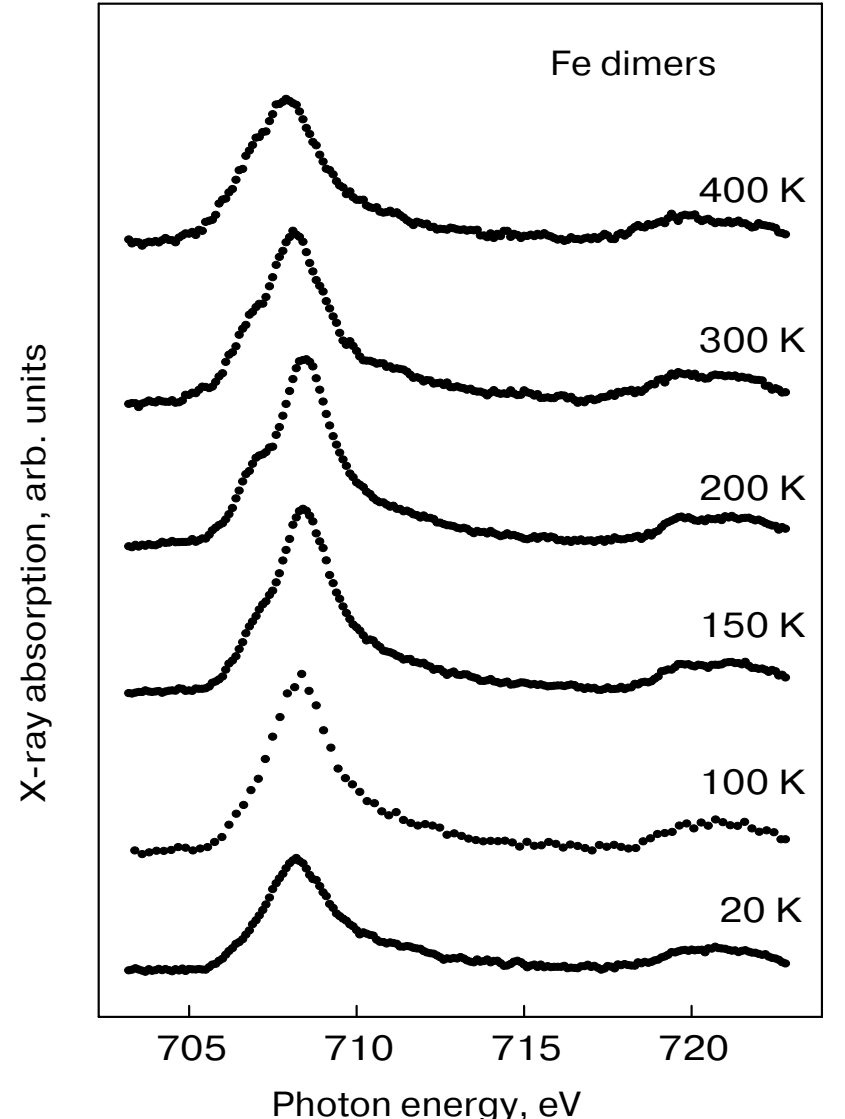

Fig. 5. $L_{2,3}$ x-ray absorption spectra of iron dimers on $(2 \times 1) \mathrm{O} / \mathrm{Ru}(001)$ for different anneal temperatures. A step edge has been subtracted from all spectra [41].

islands. Iron atoms on $(2 \times 1) \mathrm{O} / \mathrm{Ru}(001)$ seem to be highly mobile even at $150 \mathrm{~K}$.

A similar evolution of the $L_{3}$ line is shown in Fig. 5 for iron dimers on $(2 \times 1) \mathrm{O} / \mathrm{Ru}(001)$. Again, a series of $\mathrm{x}$-ray absorption spectra is shown after stepwise annealing from 20 to $400 \mathrm{~K}$. In this case, iron dimers seem to be less mobile than iron adatoms. As observed in Fig. 4 for iron adatoms, a double structure also evolves in Fig. 5 for dimers at the $L_{3}$ line after annealing to $150 \mathrm{~K}$, and is more pronounced after annealing to $200 \mathrm{~K}$. At both anneal temperatures, however, it is less pronounced than in the corresponding iron adatom spectra in Fig. 4. After annealing to 300 and $400 \mathrm{~K}$, the double structure even seems to disappear again. At $600 \mathrm{~K}$ (not shown here), only one very weak peak is visible, which has shifted further towards the position of the bulk iron line, but not reached it in energy.

Again, it can be inferred that only iron dimers are present on the $(2 \times 1) \mathrm{O} / \mathrm{Ru}(001)$ surface after deposition under soft landing conditions. However, iron dimers on $(2 \times 1) \mathrm{O} / \mathrm{Ru}(001)$ do not seem to form large islands easily. Even after annealing to 400 and $600 \mathrm{~K}$,

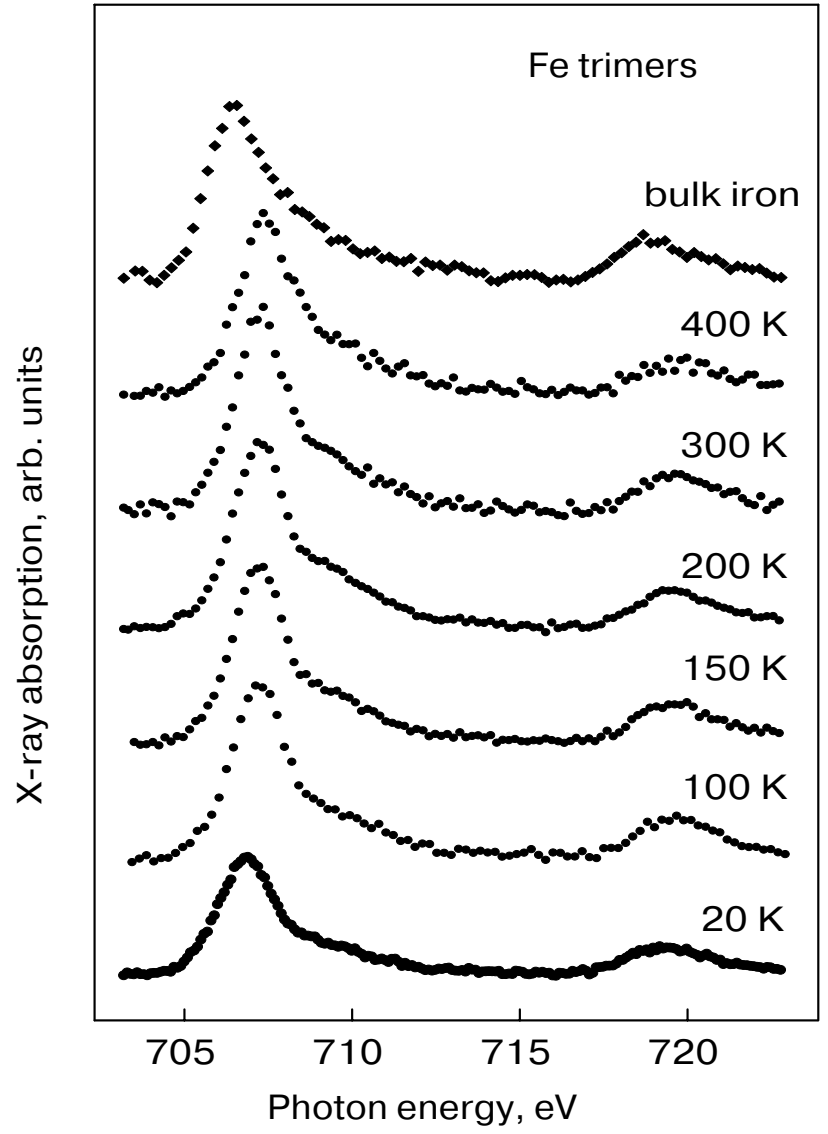

Fig. 6. $L_{2,3}$ x-ray absorption spectra of iron trimers on $(2 \times 1) \mathrm{O} / \mathrm{Ru}(001)$ for different anneal temperatures. A step edge has been subtracted from all spectra [41].

the iron dimer $L_{3}$ line is at $1 \mathrm{eV}$ higher photon energy than the corresponding bulk iron line [41].

Iron trimers on $(2 \times 1) \mathrm{O} / \mathrm{Ru}(001)$ are even more stable against diffusion than iron adatoms and dimers. This can be seen in Fig. 6, where a series of $L_{2,3}$ x-ray absorption spectra of iron trimers on $(2 \times 1) \mathrm{O} / \mathrm{Ru}(001)$ is shown. Again, soft landing conditions with argon buffer layers and deceleration of the clusters were used for trimer deposition. Upon stepwise annealing to $400 \mathrm{~K}$ there is only very little change in the iron x-ray absorption spectra. This clearly shows that almost no diffusion or agglomeration occurs with iron trimers on $(2 \times 1) \mathrm{O} / \mathrm{Ru}(001)$.

Iron adatoms, dimers, and trimers on $(2 \times 1) \mathrm{O} / \mathrm{Ru}(001)$ exhibit characteristic XAS signatures if cluster deposition is carried out with low kinetic energy into argon multilayers. In this case, soft landing is successful and only one species is present on the sample surface. In contrast, if only one monolayer of argon is used as a buffer layer, soft landing is not successful even at low kinetic energy, and fragmentation occurs as can clearly be seen in Fig. 2. 
After mass selected cluster deposition, iron adatoms, dimers, and trimers show distinctive mobilities. Annealing leads to the formation of larger island for deposited adatoms, whereas dimers and trimers on $(2 \times 1) \mathrm{O} / \mathrm{Ru}(001)$ seem to be more stable regarding diffusion and agglomeration. The discernible fingerprints of different iron clusters on $(2 \times 1) \mathrm{O} / \mathrm{Ru}(001)$ can be used to characterise soft landing conditions, at least for small clusters. Similar results have been found for small nickel clusters on $\mathrm{Ru}(001)$ and $(2 \times 1) \mathrm{O} / \mathrm{Ru}(001)[44]$.

\section{Summary}

Clusters are model systems to study the evolution of physical properties with size from single atoms to bulk matter, either on free clusters in the gas phase and on deposited clusters. To study this evolution, size selection is a prerequisite. Rare gas matrices at low temperatures can be used as a tool to accomplish nondestructive cluster deposition on surfaces. X-ray absorption spectroscopy as well as STM and optical absorption and excitation spectroscopy are well suited to study soft landing of clusters. With their characteristic XAS signatures, iron adatoms can be distinguished from dimers and trimer on $(2 \times 1) \mathrm{O} / \mathrm{Ru}(001)$ and diffusion and agglomeration can be followed by monitoring the $L_{3} \mathrm{x}$-ray absorption line.

\section{Acknowledgments}

The soft landing experiments on iron clusters reported here were performed at BESSY I and II. Technical assistance from BESSY staff members and from E20 group members at Technische Universität München is gratefully acknowledged. This project was funded by BMBF through grant KS1GUB / 5 and by DFG through grant Me 266/22-2.

1. M. Schmidt and H. Haberland, C. R. Physique 3, 327 (2002).

2. H. Kietzmann, J. Morenzin, P.S. Bechthold, G. Ganteför, and W. Eberhardt, J. Chem. Phys. 109, 2275 (1998).

3. M.-H. Schaffner, F. Patthey, U. Heiz, W.-D. Schneider, O. Kuffer, H.-V. Roy, P. Fayet, J.K. Gimzewski, and R. Berndt, Eur. Phys. J. D2, 79 (1998).

4. I.M.L. Billas, J.A. Becker, A. Châtelain, and W.A. de Heer, Phys. Rev. Lett. 71, 4067 (1993).

5. I.M.L. Billas, A. Châtelain, and W.A. de Heer, Science 265, 1682 (1994).

6. J.T. Lau, A. Föhlisch, R. Nietubyc̀, M. Reif, and W. Wurth, Phys. Rev. Lett. 89, 057201 (2002).

7. J.T. Lau, A. Föhlisch, M. Martins, R. Nietubyc̀, M. Reif, and W. Wurth, New J. Phys. 4, 98.1-98.12 (2002).
8. G. Schulze Icking-Konert, H. Handschuh, G. Ganteför, and W. Eberhardt, Phys. Rev. Lett. 76, 1047 (1996).

9. J. Ho, E.K. Parks, L. Zhu, and S.J. Riley, Chem. Phys. 201, 245 (1995).

10. L.-S. Wang, H. Wu, and S.R. Dhesi, Phys. Rev. Lett. 76, 4853 (1996).

11. H.R. Siekmann, C. Luder, J. Faehrmann, H.O. Lutz, and K.-H. Meiwes Broer, Z. Phys. D20, 410 (1991).

12. M. Gartz, C. Keutgen, S. Kuenneke, and U. Kreibig, Eur. Phys. J. D9, 127 (1999).

13. M.-H. Schaffner, J.-F. Jeannet, F. Patthey, and W.-D. Schneider, J. Phys. D: Appl. Phys. 31, 3177 (1998).

14. R. Klingeler, P.S. Bechthold, M. Neeb, and W. Eberhardt, Rev. Sci. Instrum. 73, 1803 (2002).

15. P. Jensen, Rev. Mod. Phys. 71, 1695 (1999).

16. J.A. Alonso, Chem. Rev. 100, 637 (2000).

17. C. Staudt, R. Heinrich, and A. Wucher, Nucl. Instr. and Meth. B164-165, 677 (2000).

18. J.T. Lau, Ph.D. thesis, Universität Hamburg (2002), DESY-THESIS-2002-016.

19. K. Bromann, C. Félix, H. Brune, W. Harbich, R. Monot, J. Buttet, and K. Kern, Science 274, 956 (1996).

20. W. Harbich, Philos. Mag. 79, 1307 (1999).

21. W. Harbich and C. Félix, C. R. Physique 3, 289 (2002)

22. R. Schaub, H. Jödicke, F. Brunet, R. Monot, J. Buttet, and W. Harbich, Phys. Rev. Lett. 86, 3590 (2001).

23. K. Bromann, H. Brune, C. Félix, W. Harbich, R. Monot, J. Buttet, and K. Kern, Surf. Sci. 377-379, 1051 (1997).

24. S. Fedrigo, W. Harbich, and J. Buttet, Phys. Rev. B58, 7428 (1998).

25. C. Félix, C. Sieber, W. Harbich, J. Buttet, I. Rabin, W. Schulze, and G. Ertl, Phys. Rev. Lett. 86, 2992 (2001).

26. H.-P. Cheng and U. Landman, Science 260, 1304 (1993).

27. H.-P. Cheng and U. Landman, J. Phys. Chem. 98, 352 (1994).

28. B. Nacer, C. Massobrio, and C. Félix, Phys. Rev. B56, 10590 (1997).

29. G. Vandoni, C. Félix, and C. Massobrio, Phys. Rev. B54, 1553 (1996).

30. S. Takami, K. Suzuki, M. Kubo, and A. Miyamoto, J. Nanoparticle Res. 3, 213 (2001).

31. K. Kholmurodov, I. Puzynin, W. Smith, K. Yasuoka, and T. Ebisuzaki, Comput. Phys. Commun. 141, 1 (2001).

32. M. Ratner, W. Harbich, and S. Fedrigo, Phys. Rev. B60, 11730 (1999).

33. L. Lian, C.-X. Su, and P.B. Armentrout, J. Chem. Phys. 97, 4072 (1992).

34. L. Lian, C.-X. Su, and P.B. Armentrout, J. Chem. Phys. 96, 7542 (1992).

35. S.C. Wang, U. Kürpick, and G. Ehrlich, Phys. Rev. Lett. 81, 4923 (1998). 
36. A. Golzhauser and G. Ehrlich, Phys. Rev. Lett. 77, 1334 (1996).

37. J.T. Lau, A. Achleitner, and W. Wurth, Surf. Sci. 467, 834 (2000).

38. N.A. Levanov, A.A. Katsnel'son, and A.E. Moroz, Phys. Solid State 41, 1216 (1999).

39. F.J. Palacios, M.P. Iñiguez, M.J. López, and J.A. Alonso, Phys. Rev. B60, 2908 (1999).

40. J.T. Lau, A. Achleitner, and W. Wurth, Chem. Phys. Lett. 317, 269 (2000).
41. H.-U. Ehrke, Ph.D. thesis, Technische Universität München (2000).

42. A. Wucher, N.K. Dzhemilev, I.V. Veryovkin, and S.V. Verkhoturov, Nucl. Instr. and Meth. B149, 285 (1999).

43. A. Wucher, A.D. Bekkerman, N.K. Dzhemilev, S.V. Verkhoturov, and I. Veryovkin, Nucl. Instr. and Meth. B140, 311 (1998).

44. A. Achleitner, J.T. Lau, and W. Wurth, in preparation. 